\title{
Influenza vaccination in pregnancy - current data on safety and effectiveness
}

\author{
Aneta S. Nitsch-Osuch ${ }^{1}$, Dorota Bomba-Opon² ${ }^{2}$, Mariusz Jasik ${ }^{3}$ (i) \\ ${ }^{1}$ Department of Social Medicine and Public Health, Medical University of Warsaw, Poland \\ $27^{\text {st }}$ Department of Obstetrics and Gynecology, Medical University of Warsaw, Poland \\ ${ }^{3} 2^{\text {nd }}$ Department of Obstetrics and Gynecology, Medical University of Warsaw, Poland
}

\begin{abstract}
Pregnant women are at risk of severe and complicated influenza, and so are children aged 2-5 years. Despite numerous recommendations, influenza vaccination coverage in pregnant women is still low. The trigger for this article was the development of new quadrivalent influenza vaccines along with the publication of new studies on the safety and effectiveness of inactivated influenza vaccines in pregnant women, administered also in the first trimester of pregnancy. The inactivated quadrivalent influenza vaccine is a safe and effective measure for preventing influenza in both mother and child. Live attenuated influenza vaccines are contraindicated in pregnant women, whereas inactivated influenza vaccines should be recommended to all pregnant women, either healthy or with comorbidities. Influenza vaccines can be administered during any pregnancy trimester, at least two weeks before delivery. The time of vaccination depends on vaccine availability; however, it should not be postponed unless there are significant medical contraindications.
\end{abstract}

Key words: effectiveness; influenza; pregnancy; safety; vaccine

Ginekologia Polska 2020; 91, 10: 629-633

\section{INTRODUCTION}

According to the World Health Organization (WHO), $5-10 \%$ of the adult population and $10-20 \%$ of the children population become ill with influenza seasonally, and as many as 600,000 patients die of influenza or its complications globally [1]. Groups exposed to severe and complicated influenza involve children aged $2-5$ years, senior citizens aged $>65$, patients with chronic diseases (e.g. respiratory, circulatory, nervous and metabolic diseases) and pregnant women [1]. Observations made during the $20^{\text {th }}$ century influenza pandemics show that pregnant women experience more severe influenza than the general population, and they are at a higher risk of complications, hospitalization (including intensive care treatment) and death [2]. During the Spanish flu pandemic, mortality in pregnant women was estimated at $27-45 \%$, whereas the number of deaths during the influenza pandemic in 1957 and 1968 reached 70,000 and 30,000 , respectively, whereby nearly half of the deaths were reported in pregnant women [2]. During the last influenza pandemic of 2009, the risk of influenza hospitalization in pregnant women with comorbidities was 7-8 times higher compared to the general population; the risk of complications was higher in the second and third trimester and the postpartum period (up to two weeks after the delivery), and as many as $5 \%$ of all deaths related to the influenza pandemic in the USA were reported in pregnant women [3-6].

A systematic review of 100 studies showed that the percentage of pregnant women hospitalized for influenza ranged from $5 \%$ to $87 \%, 0-22 \%$ of whom required intensive care treatment [4].

Recommendations for influenza vaccination during pregnancy were first published by the American Advisory Committee on Immunization Practices (ACIP) in 1997, and then extended in 2004 with a recommendation for influenza vaccinations in the first trimester [7]. Since 2005, the World Health Organization has been recommending vaccination for all pregnant women in a given influenza season [1]. Influenza vaccination during pregnancy is also recommended by the American College of Obstetricians and Gynecologists (ACOG) [8]. The Polish Immunization Schedule includes recommendations for influenza vaccinations in pregnant women and those planning pregnancy [9]. However, despite numerous recommendations, influenza vaccination coverage in pregnant women is still low in many low and middle income countries as well as many developed countries, 
which can be related to vaccine safety concerns of patients and medical workers, neglect of the disease severity and underestimation of the benefits of vaccination $[7,8]$.

The trigger for this publication was the development of new quadrivalent influenza vaccines along with new study results that have confirmed the effectiveness and safety of inactivated influenza vaccines in pregnant women (also in the first trimester).

\section{WHY ARE PREGNANT WOMEN EXPOSED TO SEVERE AND COMPLICATED INFLUENZA?}

Influenza incidence in pregnant women is the same as in the general population; however, disease severity and the risk of death in this group are higher [2-6]. The reason behind this phenomenon are changes in the immune system during pregnancy, such as impaired cellular immunity, which is crucial for combating viral infections [10]. Other changes that can be observed are related to humoral immunity, which is why during the influenza $A(\mathrm{H} 1 \mathrm{~N} 1)$ pandemic of 2009, pregnant women with $\mathrm{H} 1 \mathrm{~N} 1$ influenza virus infection showed lower serum lgG2 levels compared to the infected non-pregnant women, and thus they were exposed to abnormal cytokine production and more severe and complicated influenza [10]. Influenza complications during pregnancy are often related to lung function changes (reduced lung capacity and reduced tidal volume), as well as hemodynamic changes in the circulatory system (increased cardiac output, increased oxygen consumption) $[5,10]$.

\section{INFLUENZA COMPLICATIONS IN MOTHER AND CHILD}

The most frequently reported maternal influenza complications include pneumonia and acute respiratory failure, which requires respiratory support, mechanical ventilation, or even ECMO [10]. Influenza during pregnancy has been reported to increase the risk of premature delivery (with all its consequences), miscarriage, intrauterine fetal death, and low birth weight, whereas maternal fever may cause fetal tachyarrhythmias [10-14]. During the 2009 influenza pandemic, it was observed that influenza during pregnancy increased the risk of fetal death or neonatal death, as well as the likelihood of cesarean section delivery [15-17]. The tragic effects of influenza during pregnancy (including mother's or newborn's death) have also been reported in Polish scientific literature [13].

It should be emphasized that newborns and infants are at risk of severe and complicated influenza. Although influenza infections in newborns are rarely reported, recent studies have indicated vertical transmission as a route of influenza transmission, besides droplet and contact transmission [17]. In a group of children with influenza under the age of 12 months, $4 \%$ required mechanical ventilation support and $10 \%$ required intensive care treatment (hospitalization risk was higher in children aged $<3$ months as compared to those aged 6-12 months) [18].

\section{INFLUENZA PREVENTION IN PREGNANT WOMEN}

Non-specific influenza prevention measures in pregnant women include hand hygiene, the so-called "cough etiquette" and avoiding crowded places. Pre- and post-exposure pharmacological prophylaxis with neuraminidase inhibitors is also recommended $[2,4]$. The only registered drug available on the Polish market is oseltamivir for oral administration (zanamivir for inhalation is registered but not available). However, it needs to be clearly stated that oseltamivir should be primarily used for the causal treatment of influenza and must not be abused in influenza prophylaxis due to the risk of developing drug resistance, and furthermore, it can cause adverse effects (mostly associated with the digestive system) as any other drug, which is why its effectiveness in influenza prevention is estimated at $80 \%$ [19].

The most important measure of preventing influenza in pregnant women is vaccination. Currently, split vaccines and subunit vaccines are recommended in this risk group, both administered intramuscularly or subcutaneously $[7,8]$. Live attenuated influenza vaccines for intranasal administration are contraindicated during pregnancy $[7,8]$.

\section{INFLUENZA VACCINE SAFETY IN PREGNANCY}

Data on inactivated vaccines for seasonal influenza indicate that they are safe for both mother and child. A retrospective observational study by Nordin et al., conducted on a group 79,900 vaccinated and 148,000 unvaccinated pregnant women, showed that there was no increased risk of adverse vaccination reactions within 42 days after the vaccination [20]. Approximately 2.4 million pregnant women were vaccinated against influenza in the US in the 20092010 season, and no increased risk to mother or child was shown [20]. A detailed systematic review on influenza vaccine safety in pregnancy showed that inactivated influenza vaccines do not increase the risk of intrauterine fetal death, spontaneous miscarriage or the occurrence of birth defects in a child [21]. Data on the safety of influenza vaccines in pregnancy refer to inactivated vaccines with split virion or sub-unit vaccines without adjuvants (these vaccines are available in Poland). Currently, there is not enough data on the safety of adjuvanted vaccine, such as MF59 (squalene oil-based adjuvant used since 1997) and ASO3 (a combination of tocopherol and squalene in an oil-in-water emulsion used during the 2009 pandemic), so as to recommend them for pregnant women $[7,8]$. One retrospective study conducted on a group of pregnant women showed a higher incidence of gestational diabetes and eclampsia among 
women who were administered an MF59 adjuvanted influenza vaccine [22]. In a randomized, controlled clinical trial comparing the MF59-adjuvanted vaccine in pregnant and non-pregnant women, a negligible tendency for a lower immune response was observed in pregnant women; $72 \%$ of women reported adverse reactions, $64 \%$ had local reactions and $26 \%$ had systemic reactions, the most common symptom being malaise [23]. AS03-adjuvanted influenza vaccine, which was administered to approximately 380,000 pregnant women, was associated with an increased risk of narcolepsy in children, which shows that oil emulsion-based adjuvants are not fully safe in less studied patient populations, such as children and pregnant women [24].

The number of studies on the effectiveness of vaccines based on recombinant hemagglutinin protein that is produced in insect cells (intended for American adults allergic to chicken egg protein) is also insufficient so as to recommend these vaccines for pregnant women [3].

Studies performed after the 2009 influenza pandemic have confirmed the safety of inactivated vaccines against influenza in pregnant women. In a randomized trial from the USA, Munoz et al. proved that two inactivated trivalent seasonal influenza vaccines are safe [21]. In an Australian cohort, no evidence on the increased occurrence of adverse reactions was found in vaccinated pregnant women as compared to non-pregnant women [25].

Recently, increasingly more scientific reports proving influenza vaccine safety in the first trimester have been published.

The effect of influenza vaccination on pregnancy and fetal development was assessed by Japanese researchers in the $2013 / 2014$ season [26]. The following endpoints were considered: miscarriage, fetal death, preterm delivery, low birth weight and birth defects. Adverse delivery outcomes were reported for 641 (10\%) of 6,387 unvaccinated pregnant women and 356 (9\%) of 3,943 vaccinated pregnant women. Even considering potential confounding factors, vaccinations during pregnancy were not associated with a risk of adverse delivery outcomes (odds ratio $0.90,95 \%$ confidence interval 0.76-1.07). Vaccinations in the first or second trimester showed no association with adverse delivery outcomes, while vaccination in the third trimester was associated with a reduced risk of adverse delivery outcomes (odds ratio $0.70,95 \%$ confidence interval $0.51-0.98$ ) [26]. Similar conclusions regarding the performance of vaccinations in the first trimester were also made by American researchers in a study from 2004-2013 [27]. A total of 52,856 children of vaccinated mothers were assessed with regard to their psychomotor development in the first 12 months of life and compared with 373,088 children of mothers who were not vaccinated in the first trimester. The incidence of structural birth defects (per 100 live births) was 1.6 in the group of children of vaccinated mothers compared to 1.5 in the group of children of unvaccinated mothers (corrected PR of 1.02, 95\% Cl 0.94-1.10) [27].

\section{EFFECTIVENESS OF INFLUENZA VACCINATIONS IN PREGNANCY}

The immunogenicity of influenza vaccines in pregnant women is comparable to that observed in non-pregnant women (i.e. the post-vaccination level of antibodies is comparable). Similar results were observed in the 1970s in the studies on A/New Jersey/8/76 monovalent vaccine, which showed no reduction in the number of vaccine antibodies in pregnant women compared to the general population [2]. During the 2009 pandemic, it was confirmed that the inactivated monovalent $\mathrm{H} 1 \mathrm{~N} 1 / 2009 \mathrm{pdm}$ vaccine was immunogenic in pregnant women, although women who had previously been administered a seasonal vaccine showed a lower seroprotection rate [28].

The actual effectiveness of influenza vaccines during pregnancy has also been proven, along with vaccination benefits for both mother and child.

A randomized clinical trial entitled "Mother's Gift", which was conducted in Bangladesh by Zamana et al. [29] in 2004-2005, focused on the effectiveness of influenza vaccinations in the third trimester and the obtained results were compared with the numbers related to pneumococcal polysaccharide vaccinations (control group) [29]. It was found that the number of laboratory confirmed influenza cases decreased by $63 \%$ in children of mothers who were vaccinated during pregnancy (this effect was observed during the first 6 months of the child's life) [29]. Vaccinations in the third trimester resulted in a reduced number of infections with fever by $29 \%$ in infants and $36 \%$ in mothers [29].

Protective antibody levels were maintained in infants for 6 months, with an estimated half-life of 42-50 days. A higher level of IgA antibodies was also found in the breast milk of influenza-vaccinated pregnant mothers for 6 months [29].

A clinical study conducted by Thompson et al. [30] in two influenza seasons 2010-2012 showed that influenza vaccinations in pregnant women reduced the risk of acute respiratory symptoms associated with laboratory-confirmed influenza by approx 50\%.

A quadrivalent influenza vaccine has been available since 2012, and it has replaced the trivalent vaccine. This vaccine contains two influenza A virus subtypes and two currently circulating influenza B virus strains (Victoria and Yamagata), whereas the trivalent vaccine contains only one of the influenza $B$ virus strains. To assess the actual effectiveness of the quadrivalent inactivated split vaccine, a randomized, actively controlled blinded study on a group of 4,193 pregnant women in the third trimester was conducted in Mali from September 2011 to April 2013 [31]. The study involved 
weekly medical visits, during which infants up to 6 months of age were tested for an influenza-like disease, and RT-PCR tests were made to identify laboratory-confirmed influenza [31]. The primary objectives of the study included: 1 . Assessment of the effectiveness of the vaccine against the first laboratory-confirmed influenza episode in infants born to women vaccinated at any time before delivery; 2 . Assessment of the effectiveness of the vaccine against the first laboratory-confirmed influenza episode in infants born to women vaccinated $\geq 14$ days before delivery. The secondary purpose of the study was assessment of the effectiveness of the vaccine against the first laboratory-confirmed influenza episode in women (before and after delivery) [31]. Infants aged < 6 months, whose mothers had been vaccinated at any time during pregnancy, showed vaccination effectiveness of $33.1 \%$ (95\% Cl 3.7-53.9\%), whereby the effectiveness against laboratory-confirmed influenza in the first 4 months of life was $67.9 \%$ (95\% Cl 35.1-85.3\%). Infants $<6$ months whose mothers were vaccinated $\geq 14$ days before the delivery showed vaccine effectiveness of $37.3 \%$ (95\% Cl 7.6-57.8\%); however, during the first 4 months of life the effectiveness was higher and reached $70.2 \%(95 \% \mathrm{Cl}$ 35.7-87.6\%). Vaccine effectiveness in preventing laboratory-confirmed influenza in vaccinated women was estimated at $70.3 \%$ (95\% Cl $42.2-85.8 \%)$ [31].

The benefits of vaccinating pregnant women can be observed in the first six months of child's life. Since the influenza vaccine can be administered from the age of six months, infants cannot be vaccinated. Due to the functional immaturity of their immune system, infants, especially those aged $>6$ months, suffer from influenza more frequently and are five times more likely to be hospitalized, as compared to infants aged 6-23 months [22].

In the light of the above statistics, a mother's vaccination protects a child who can become ill with influenza but is too young to be vaccinated. Vaccination results in the production of maternal antibodies which are transmitted to the child transplacentally and in breast milk. The degree and duration of the child protection are related to the level of maternal influenza antibody titers, which in turn depends on the time interval between vaccination and delivery [32]. How long the passively acquired antibodies persist in infants, it depends on their initial concentration in the umbilical cord blood; however, it is no longer than 6 months [32]. The effect of a mother's vaccination on a child has been evaluated in many studies. In a controlled comparative study from 2000-2009 (before H1N1 2009 pandemic), there were $90 \%$ fewer influenza-related hospitalizations in a group of infants aged $<6$ months and born to influenza vaccinated mothers than in the group of children of non-vaccinated mothers [33]. Since no seroprotection was observed in children of mothers who had been vaccinated only 15 days before delivery, it can be assumed that vaccination against influenza should be performed at least 2 weeks before delivery to maximize the neonatal protection [33]. In a UK study, a mother's vaccination during pregnancy in the $2013 / 2014$ season was effective for $71 \%$ infants under 6 months of age [34].

\section{WHEN TO GET VACCINATED?}

Influenza vaccination with an inactivated vaccine can be performed in any pregnancy trimester. In order to obtain optimal protection against influenza in both mother and child, it is recommended to perform a vaccination at least 2 weeks before the planned delivery. Later vaccination is safe but might not be effective enough (time between the production of antibodies and their transplacental transfer is too short). However, it should be emphasized that vaccination in this time is still beneficial and so is vaccination in the postpartum period, as part of the cocoon strategy. The strategy involves vaccinating people in the immediate environment of a patient (the newborn) who may become ill but cannot be vaccinated (because of being too young). Being part of the cocoon strategy, vaccinations in the immediate environment reduce the transmission of pathogens and the risk of becoming ill [35].

For many years, influenza vaccinations were recommended only when the vaccine composition recommended for a given season was available (most often in September). However, it should be emphasized that influenza vaccination can and should be performed during any autumn or winter month when there is increased influenza virus circulation. Postponement of the vaccination, caused e.g. by a delay in the vaccine development (which took place previously and was caused e.g. by the delay in the publication of the WHO vaccine composition recommendations for a given season or technological/production difficulties) does not adversely affect the effectiveness of vaccination. Furthermore, it can have a positive aspect. Antibodies have been shown to persist for up to 6 months and gradually disappear after the vaccination, which is why early vaccination may result in low levels of antibodies at the end of the influenza season [35]. In the case of patients at risk (e.g. patients with chronic respiratory, circulatory and metabolic diseases), vaccination is currently not recommended at the beginning of the season but later to ensure high antibody level throughout the influenza season [35].

\section{CONCLUSIONS}

Inactivated quadrivalent influenza vaccine is a safe and effective measure for preventing influenza in mothers and children. It can be recommended to all pregnant women, who are healthy or have comorbidities. The vaccine can be administered in any pregnancy trimester. The time of vac- 
cination depends on the vaccine availability; however, it should not be postponed unless there are significant medical counterindications.

\section{Conflict of interest}

The authors declare no conflict of interests.

\section{REFERENCES}

1. World Health Organization (WHO). Influenza seasonal . https:// www.who.int/en/news-room/fact-sheets/detail/influenza-(seasonal) (11.04.2020).

2. Kourtis AP, Read JS, Jamieson DJ. Pregnancy and infection. N Engl J Med. 2014; 370(23): 2211-2218, doi: 10.1056/NEJMra1213566, indexed in Pubmed: 24897084.

3. Siston AM, Rasmussen SA, Honein MA, et al. Pandemic H1N1 Influenza in Pregnancy Working Group. Pandemic 2009 influenza A(H1 N1) virus illness among pregnant women in the United States. JAMA. 2010; 303(15): 1517-1525, doi: 10.1001/jama.2010.479, indexed in Pubmed: 20407061.

4. Meijer WJ, van Noortwijk AGA, Bruinse HW, et al. Influenza virus infection in pregnancy: a review. Acta Obstet Gynecol Scand. 2015; 94(8): 797-819, doi: 10.1111/aogs.12680, indexed in Pubmed: 26012384.

5. Neuzil KM, Reed GW, Mitchel EF, et al. Impact of influenza on acute cardiopulmonary hospitalizations in pregnant women. Am J Epidemiol. 1998; 148(11): 1094-1102, doi: 10.1093/oxfordjournals.aje.a009587, indexed in Pubmed: 9850132

6. Mazagatos C, Delgado-Sanz C, Oliva J, et al. Spanish Influenza Surveillance System. Exploring the risk of severe outcomes and the role of seasonal influenza vaccination in pregnant women hospitalized with confirmed influenza, Spain, 2010/11-2015/16. PLoS One. 2018; 13(8): e0200934, doi: 10.1371/journal.pone.0200934, indexed in Pubmed: 30089148.

7. Centers for Diseases Control and Prevention (CDC). 2010. Prevention and control of influenza with vaccines: recommendations of the Advisory Committee on Immunization Practices (ACIP) 2010. MMWR. 2010; 59 (RR-8): 46.

8. ACOG Committee Opinion \#305: Influenza Vaccination and Treatment During Pregnancy. Obstetrics \& Gynecology. 2004; 104(5, Part 1): 1125-1126, doi: 10.1097/00006250-200411000-00058.

9. Komunikat Głównego Inspektora Sanitarnego z dnia 16 października 2019 r. w sprawie programu szczepień ochronnych na rok 2020. http:// dziennikmz.mz.gov.pl/api/DUM_MZ/2019/87/journal/5727 (24.4.2020).

10. Sappenfield E, Jamieson DJ, Kourtis AP. Pregnancy and susceptibility to infectious diseases. Infect Dis Obstet Gynecol. 2013; 2013: 752852, doi: 10.1155/2013/752852, indexed in Pubmed: 23935259.

11. Creanga AA, Johnson TF, Graitcer SB, et al. Severity of 2009 pandemic influenza $A(\mathrm{H} 1 \mathrm{~N} 1)$ virus infection in pregnant women. Obstet Gynecol. 2010; 115(4): 717-726, doi: 10.1097/AOG.0b013e3181d57947, indexed in Pubmed: 20308830.

12. Håberg SE, Trogstad L, Gunnes N, et al. Risk of fetal death after pandemic influenza virus infection or vaccination. N Engl J Med. 2013; 368(4): 333-340, doi: 10.1056/NEJMoa1207210, indexed in Pubmed: 23323868.

13. Piechota $Z$, Botiuk K, Skręt A. Severe A/H1N1 influenza in four pregnant women in Podkarpacie Province of Poland. Ginekol Pol. 2010; 81(3): 227-231.

14. Hamela-Olkowska A, Szymkiewicz-Dangel J. Tachyarytmie u płodów - aktualny stan wiedzy. Ginekol Pol. 2010; 81: 844-850.

15. Stanwell-Smith R, Parker AM, Chakraverty $P$, et al. Possible association of influenza A with fetal loss: investigation of a cluster of spontaneous abortions and stillbirths. Commun Dis Rep CDR Rev. 1994; 4(3): R28-R32, indexed in Pubmed: 7513232

16. Håberg SE, Trogstad L, Gunnes N, et al. Risk of fetal death after pandemic influenza virus infection or vaccination. N Engl J Med. 2013; 368(4): 333-340, doi: 10.1056/NEJMoa1207210, indexed in Pubmed: 23323868.

17. Mosby LG, Rasmussen SA, Jamieson DJ. 2009 pandemic influenza $A(H 1 N 1)$ in pregnancy: a systematic review of the literature. Am J Obstet Gynecol. 2011; 205(1): 10-18, doi: 10.1016/j.ajog.2010.12.033, indexed in Pubmed: 21345415.

18. Chaves SS, Perez A, Farley MM, et al. Influenza Hospitalization Surveillance Network. The burden of influenza hospitalizations in infants from
2003 to 2012, United States. Pediatr Infect Dis J. 2014; 33(9): 912-919, doi: 10.1097/INF.0000000000000321, indexed in Pubmed: 24577042.

19. Brydak LB, Nitsch-Osuch A, Nitsch-Osuch A, et al. [Vaccination against influenza in pregnant women - safety and effectiveness]. Ginekol Pol. 2013; 84(1): 56-61, doi: 10.17772/gp/1541, indexed in Pubmed: 23488311.

20. Nordin JD, Kharbanda EO, Benitez GV, et al. Maternal safety of trivalent inactivated influenza vaccine in pregnant women. Obstet Gynecol. 2013; 121(3): 519-525, doi: 10.1097/AOG.0b013e3182831b83, indexed in Pubmed: 23635613.

21. Munoz FM, Jackson LA, Swamy GK, et al. Safety and immunogenicity of seasonal trivalent inactivated influenza vaccines in pregnant women. Vaccine. 2018; 36(52): 8054-8061, doi: 10.1016/j.vaccine.2018.10.088, indexed in Pubmed: 30416018.

22. McMillan M, Porritt $K$, Kralik D, et al. Influenza vaccination during pregnancy: a systematic review of fetal death, spontaneous abortion, and congenital malformation safety outcomes. Vaccine. 2015; 33(18): 21082117, doi: 10.1016/j.vaccine.2015.02.068, indexed in Pubmed: 25758932.

23. Bischoff AL, Følsgaard NV, Carson CG, et al. Altered response to $A(H 1 N 1)$ pnd09 vaccination in pregnant women: a single blinded randomized controlled trial. PLoS One. 2013; 8(4): e56700, doi: 10.1371/journal. pone.0056700, indexed in Pubmed: 23637733.

24. Partinen $M$, Saarenpää-Heikkilä O, Ilveskoski I, et al. Increased incidence and clinical picture of childhood narcolepsy following the $2009 \mathrm{H} 1 \mathrm{~N} 1$ pandemic vaccination campaign in Finland. PLoS One. 2012; 7(3): e33723, doi: 10.1371/journal.pone.0033723, indexed in Pubmed: 22470463.

25. Regan AK, Tracey L, Blyth CC, et al. A prospective cohort study comparing the reactogenicity of trivalent influenza vaccine in pregnant and non-pregnant women. BMC Pregnancy Childbirth. 2015; 15: 61, doi: 10.1186/s12884-015-0495-2, indexed in Pubmed: 25880741.

26. Ohfuji S, Deguchi M, Tachibana D, et al. Osaka Pregnant Women Influenza Study Group. Safety of influenza vaccination on adverse birth outcomes among pregnant women: A prospective cohort study in Japan. Int J Infect Dis. 2020; 93: 68-76, doi: 10.1016/j.ijid.2020.01.033, indexed in Pubmed: 31982621.

27. Kharbanda EO, Vazquez-Benitez G, Romitti PA, et al. Vaccine safety datalink. First trimester influenza vaccination and risks for major structural birth defects in offspring. J Pediatr. 2017; 187: 234-239.e4, doi: 10.1016/j. jpeds.2017.04.039, indexed in Pubmed: 28550954.

28. Ohfuji S, Fukushima W, Deguchi M, et al. Immunogenicity of a monovalent 2009 influenza $A$ ( $\mathrm{H} 1 \mathrm{~N} 1$ ) vaccine among pregnant women: lowered antibody response by prior seasonal vaccination. J Infect Dis. 2011;203(9): 1301-1308, doi: 10.1093/infdis/jir026, indexed in Pubmed: 21459817.

29. Zaman $\mathrm{K}$, Roy $\mathrm{E}$, Arifeen $\mathrm{SE}$, et al. Effectiveness of maternal influenza immunization in mothers and infants. N Engl J Med. 2008; 359(15): $1555-$ 1564, doi: 10.1056/NEJMoa0708630, indexed in Pubmed: 18799552.

30. Thompson MG, Li DK, Shifflett $P$, et al. Pregnancy and Influenza Project Workgroup. Effectiveness of seasonal trivalent influenza vaccine for preventing influenza virus illness among pregnant women: a population-based case-control study during the 2010-2011 and 2011-2012 influenza seasons. Clin Infect Dis. 2014; 58(4): 449-457, doi: 10.1093/cid/cit750, indexed in Pubmed: 24280090.

31. Tapia MD, Sow SO, Tamboura B, et al. Maternal immunisation with trivalent inactivated influenza vaccine for prevention of influenza in infants in Mali: a prospective, active-controlled, observer-blind, randomised phase 4 trial. Lancet Infect Dis. 2016; 16(9): 1026-1035, doi: 10.1016/S14733099(16)30054-8, indexed in Pubmed: 27261067.

32. Poehling KA, Edwards KM, Weinberg GA, et al. New Vaccine Surveillance Network. The underrecognized burden of influenza in young children. $\mathrm{N}$ Engl J Med. 2006; 355(1): 31-40, doi: 10.1056/NEJMoa054869, indexed in Pubmed: 16822994

33. Benowitz I, Esposito DB, Gracey KD, et al. Influenza vaccine given to pregnant women reduces hospitalization due to influenza in their infants. Clin Infect Dis. 2010; 51(12): 1355-1361, doi: 10.1086/657309, indexed in Pubmed: 21058908.

34. Dabrera $\mathrm{G}$, Zhao $\mathrm{H}$, Andrews $\mathrm{N}$, et al. Effectiveness of seasonal influenza vaccination during pregnancy in preventing influenza infection in infants, England, 2013/14. Euro Surveill. 2014; 19(45): 20959, doi: 10.2807/1560-7917.es2014.19.45.20959, indexed in Pubmed: 25411687.

35. Newall AT, Chen C, Wood JG, et al. Within-season influenza vaccine waning suggests potential net benefits to delayed vaccination in older adults in the United States. Vaccine. 2018; 36(39): 5910-5915, doi: 10.1016/j. vaccine.2018.08.007, indexed in Pubmed: 30146403. 